\title{
A New Numerical Algorithm of Three Parameter Generalized Extreme Value Distribution of Wind Speed
}

\author{
$\mathrm{Ye} \mathrm{Tao}{ }^{1,2}$ \\ ${ }^{1}$ State Key Laboratory for Disaster Mitigation in Civil Engineering, Tongji University, Shanghai 200092, China; \\ ${ }^{2}$ Department of Bridge Engineering, Tongji University, Shanghai 200092, China
}

\begin{abstract}
Maximum likelihood estimation method is used to solve the problem of parameter estimation of three-parameter generalized extreme value distribution. Based on the theory of order reducing, a new numerical algorithm is presented to resolve the problem of maximum likelihood estimation of threeparameter generalized extreme value distribution. Firstly, the shape parameter is assumed to be known and ternary likelihood equations can be transferred into binary ones that are solved with the dichotomy. And then, scale and location parameters are the functions of shape parameter. Further, the maximum likelihood function is described as a unitary function of shape parameter. The optimal estimation of shape parameters can be obtained by applying dichotomy again.
\end{abstract}

\section{Introduction}

Wind disaster is one of the most serious natural disasters in the world. In order to effectively avoid wind disaster, the determination of wind load plays an important role in building structure design. Therefore, the accurate estimation of extreme wind speed is very important for the determination of wind load. At present, the wind load code [1] can only be used for the static analysis of wind load on general structures; for the dynamic and reliability analysis of wind load on important structures, it is necessary to use the original wind speed records of adjacent wind speed stations on the project site. For the mean wind, there are three main methods to estimate the probability of maximum wind action. The first method is based on the theory of stochastic process, which can reflect the double randomness of mean wind time and space. Limited to the current technical level, the research of this method can not be used in practice; the second method is called annual maximum wind action time series method, which is suitable for structural design with less obvious aeroelastic effect; the third method is based on the joint distribution of wind speed and direction [2]. The mean wind is independently described as a random variable model in different directions. This method can be used not only for rigid structures, but also for structures or components with wind-induced aerodynamic amplification or aeroelastic effects.

At present, the joint distribution probability model is widely used. Scholars at home and abroad have done a lot of research work on the calculation of extreme wind speed [3] [4] [5] [6]. The calculation of extreme wind speed mainly comes from three aspects of error, namely, sample sampling, model selection and parameter estimation. Therefore, many research results are not the same [7] [8] [9] [10] [11], and the wind speed distribution probabilities adopted by national standards are also different. Parameter estimation is one of the core problems of extreme value distribution model. The commonly used methods include maximum likelihood method, moment method, probability weighted moment method and regression method. Maximum likelihood estimation is the most basic method, which makes the results of parameter estimation reflect the statistical information of the sample as a whole and has good statistical properties, so it is widely used. However, in this process, nonlinear equations need to be solved numerically, so the calculation amount is large and it is not convenient to apply; moment method and probability weighted moment method use the digital characteristic "moment" of the sample to estimate the digital characteristics of the population, and the calculation is relatively simple; regression method uses linear or nonlinear regression to obtain the estimation of the distribution parameters after the initial value of the parameters is given.

In this paper, a new numerical method is proposed for the maximum likelihood equations of three parameter extreme wind speed distribution parameters by combining the order reduction method with the dichotomy method. This method doesn't need to give initial value, and it's simple and fast, and it's not limited by the number of samples. Based on the wind speed data of Baoshan meteorological station in Shanghai, this paper discusses the solution strategy of the nonlinear equation of extreme value distribution by combining with the maximum likelihood method, and establishes an efficient and strong stability analysis method step by step iterative estimation method. Taking the wind speed statistical analysis of Baoshan meteorological station in

\footnotetext{
* Corresponding author: 403197915@qq.com
} 
Shanghai as an example, the above theory and method are applied to engineering practice.

The three-parameter extreme value distribution is a relatively perfect distribution, which is flexible in fitting random data and adaptable to frequency distributions of different shapes. When different values are taken as the shape parameters take, it can be equivalent to or close to some other commonly used distributions [12]. However, the parameter estimation of three parameter extreme value distribution is more complex. Research work was carried out on this topic and put forward many methods. At present, the common estimation methods are graph parameter estimation, moment estimation, maximum likelihood estimation and so on. The graph parameter estimation method is easy to use, intuitive and easy to understand, and also suitable for truncated life test. However, this method relies on the visual review of data curve, has strict requirements for drawing quality and large deviation of estimation results [13]. The moment estimation method first calculates the standard deviation, mean value and skewness coefficient of the sample, and then uses the ready-made number table to estimate the distribution parameters. Some scholars, such as bartolucci, Ahmad, Fu Huimin, have also proposed some improved algorithms, but the results of these methods are still not satisfactory [14] [15] [16].

How to estimate the parameters of three parameter generalized extreme value distribution from samples is an important problem in engineering. The common methods are maximum likelihood method and graph estimation method. The traditional maximum likelihood method has high accuracy and is a very effective and general parameter estimation method, but it is very complex to use iterative method to solve three simultaneous transcendental equations. In addition, many excellent properties of the maximum likelihood method are not tenable because they sometimes do not meet the normal regularization conditions, resulting in the maximum likelihood estimation does not exist or has multiple solutions [17]. For this reason, people have been discussing the parameter estimation of three parameter generalized extreme value distribution, and put forward various more reasonable and simple methods. Qi et al first used Newton Raphson method to obtain the maximum likelihood estimation of two parameter extreme value distribution, and then gradually solved the parameter estimation of three parameter extreme value distribution [18]. Gove is solved by nonlinear optimization method [19]. In 1987, Qu Yanlu et al. Proposed a reduced order likelihood method to solve the equations [20]. Wang Huasheng et al. Aimed at maximizing the likelihood function value and found the best location parameter with a certain step size. When the location parameter was determined, the likelihood equations degenerated into two-dimensional equations [21]. Although these methods are simple and intuitive, the accuracy is not ideal, the amount of calculation is large, and the influence of initial value is obvious. The probability weighted moment method is also a parameter estimation method. This method uses the probability weighted moment of the sample to estimate the probability weighted value of the population to obtain the parameter estimation [22].

\section{Maximum likelihood estimation of extreme distribution parameters}

\subsection{Probability model of extreme wind speed}

If the wind speed stochastic process is regarded as a stationary process, then the extreme wind speed has an accurate distribution in theory. However, the actual wind speed time history is not a strictly stationary process, which leads to the existence of errors. The error of probability analysis of extreme wind speed mainly comes from three aspects: sample selection, probability distribution model selection and parameter estimation. To determine the optimal extreme value distribution model, we should start from two aspects. On the one hand, we should collect and use more abundant and accurate original data of wind speed; on the other hand, we should improve the accuracy of statistical methods. The analysis method should adopt the maximum likelihood method and other parameter estimation methods with higher accuracy than the moment method, least square method and other simplified methods of extreme value parameters.

The extreme wind speed can be fitted by different probability distribution models. For example, if the value of random variable is expressed, the distribution functions of extreme wind speed are Gumbel distribution, Frechet distribution and Weibull distribution

I: Gumbel distribution

$$
F_{G \theta}(x)=\exp \left[-\exp \left(-\frac{x-\mu(\theta)}{\sigma(\theta)}\right)\right]
$$

II: Frechet distribution

$$
F_{F \theta}(x)=\exp \left[-\left(\frac{x-\mu(\theta)}{\sigma(\theta)}\right)^{-K(\theta)}\right]
$$

III: Weibull distribution

$$
F_{W \theta}(x)=\exp \left[-\left(-\frac{x-\mu(\theta)}{\sigma(\theta)}\right)^{K(\theta)}\right]
$$

\subsection{Maximum likelihood equations}

The basic idea of maximum likelihood estimation is to select the undetermined parameter to maximize the probability of the sample appearing in the field of the observation value, and take this value as the point estimation value of the unknown parameter [21]. Maximum likelihood estimation is a very effective and general parameter estimation method, which plays an important role in parameter estimation. Maximum likelihood estimation has obvious advantages. However, in the estimation of three parameter generalized extreme value distribution, this method needs more calculation and it is time-consuming. In application, Based on the maximum likelihood function, people often use other methods to analyze the three parameter generalized extreme value distribution. This method not only ensures 
the accuracy of maximum likelihood estimation, but also avoids complicated calculation.

Take Weibull distribution as an example to illustrate the algorithm in this paper:

$$
f_{X}(x)=\frac{K}{\sigma}\left(-\frac{x-\mu}{\sigma}\right)^{K-1} \cdot \exp \left[-\left(-\frac{x-\mu}{\sigma}\right)^{K}\right], x \leq \mu
$$

So the likelihood function of the sample is:

$$
\begin{aligned}
& L\left(x_{1}, x_{2}, \cdots x_{n}, K, \sigma, \mu\right)=\prod_{i=1}^{n} f_{X}\left(x_{i}, K, \sigma, \mu\right) \\
& =\frac{K^{n}}{\sigma^{K n}} \prod_{i=1}^{n}\left(\mu-x_{i}\right)^{(K-1)} \exp \left[-\sum_{i=1}^{n}\left(\frac{\mu-x_{i}}{\sigma}\right)^{K}\right]
\end{aligned}
$$

The log likelihood function is:

$$
\begin{aligned}
& \ln L\left(x_{1}, x_{2}, \cdots x_{n}, K, \sigma, \mu\right)=\ln \left[\prod_{i=1}^{n} f_{X}\left(x_{i}, K, \sigma, \mu\right)\right] \\
& =n \ln K-K n \ln \sigma+(K-1) \sum_{i=1}^{n} \ln \left(\mu-x_{i}\right)-\sum_{i=1}^{n}\left(\frac{\mu-x_{i}}{\sigma}\right)^{K}
\end{aligned}
$$

here $K, \sigma$ and $\mu$ are the shape parameter, scale parameter and position parameter of the three parameter Weibull distribution, and $n$ is the number of samples $x_{i}$. When there are triple values $\left(K^{*}, \sigma^{*}, \mu^{*}\right)$ satisfying:

$$
\begin{aligned}
& \text { or } L\left(K^{*}, \sigma^{*}, \mu^{*}\right)=\max \left[L\left(x_{1}, x_{2}, \cdots x_{n}, K, \sigma, \mu\right)\right] \\
& \ln L\left(K^{*}, \sigma^{*}, \mu^{*}\right)=\max \left[\ln L\left(x_{1}, x_{2}, \cdots x_{n}, K, \sigma, \mu\right)\right]
\end{aligned}
$$

$\left(K^{*}, \sigma^{*}, \mu^{*}\right)$ is considered to be the maximum

likelihood estimator of $K, \sigma, \mu$ respectively.

Since the maximum likelihood estimator of the distribution parameter is the maximum point of the maximum likelihood function, when the likelihood function is a continuously differentiable function of the distribution parameter and the maximum point is the interior point of the parameter interval, the maximum likelihood estimator of the distribution parameter is the solution of the following equations (8).

$$
\left\{\begin{array}{l}
\frac{\partial \ln L\left(x_{1}, x_{2}, \cdots x_{n}, K, \sigma, \mu\right)}{\partial K}=\frac{n}{K}+ \\
\frac{\sum_{i=1}^{n} \ln \frac{\mu-x_{i}}{\sigma}-\sum_{i=1}^{n}\left\{\left(\frac{\mu-x_{i}}{\sigma}\right)^{K} \ln \frac{\mu-x_{i}}{\sigma}\right\}=0}{\frac{\partial \ln L\left(x_{1}, x_{2}, \cdots x_{n}, K, \sigma, \mu\right)}{\partial \sigma}=n-\frac{1}{\sigma^{K}} \sum_{i=1}^{n}\left(\mu-x_{i}\right)^{K}=0} \\
\frac{\partial \ln L\left(x_{1}, x_{2}, \cdots x_{n}, K, \sigma, \mu\right)}{\partial \mu}= \\
(K-1) \sum_{i=1}^{n}\left(\mu-x_{i}\right)^{-1}-\frac{K}{\sigma} \sum_{i=1}^{n}\left(\frac{\mu-x_{i}}{\sigma}\right)^{K-1}=0
\end{array}\right.
$$

In this paper, a numerical solution is proposed for the equations (8), which is introduced below.

\subsection{Numerical solution of maximum likelihood equations}

Practical application shows that the parameter convergence domain of equation group (8) is very small, and the given initial value is usually required to be very close to the true solution. Obviously, it is difficult to achieve this in practical calculation [11]. If one of the three parameters is determined, the equations (8) will degenerate into a system of binary equations. Thus, the other two parameters can be determined according to the numerical analysis method. Assuming that shape parameter $K$ has been determined, then the scale parameter $\sigma$ and position parameter $\mu$ can be determined by the equation group (9).

$$
\left\{\begin{array}{l}
n-\frac{1}{\sigma^{K}} \sum_{i=1}^{n}\left(\mu-x_{i}\right)^{K}=0 \\
(K-1) \sum_{i=1}^{n}\left(\mu-x_{i}\right)^{-1}-\frac{K}{\sigma} \sum_{i=1}^{n}\left(\frac{\mu-x_{i}}{\sigma}\right)^{K-1}=0
\end{array}\right.
$$

If the scale parameter $\sigma$ in the system of equations (9) is eliminated, it is further reduced to a univariate equation

$$
\frac{K-1}{n K} \sum_{i=1}^{n}\left(\mu-x_{i}\right)^{-1} \sum_{i=1}^{n}\left(\mu-x_{i}\right)^{K}-\sum_{i=1}^{n}\left(\mu-x_{i}\right)^{K-1}=0
$$

When $K>1$, equation (11) is a function of the position parameter.

$$
y=\frac{K-1}{n K} \sum_{i=1}^{n}\left(\mu-x_{i}\right)^{-1} \sum_{i=1}^{n}\left(\mu-x_{i}\right)^{K}-\sum_{i=1}^{n}\left(\mu-x_{i}\right)^{K-1}
$$

In the interval $\mu \in\left(0, \min \left\{x_{i}\right\}\right)$, when the shape parameter $K$ is given, it is easy to find the zero point of equation (11) by dichotomy, that is, the position parameter $\mu$, which is unique. The analytic solution of scale parameter $\sigma$ can be obtained by substituting $\mu$ into the first formula of equation group (9).

$$
\sigma=\left[\frac{1}{n} \sum_{i=1}^{n}\left(\mu-x_{i}\right)^{K}\right]^{\frac{1}{K}}
$$

It can be seen from the mentioned above that each shape parameter $K$ corresponds to a unique position parameter $\mu$ and a scale parameter $\sigma$. Therefore, we can regard $\mu$ and $\sigma$ as a function of $K$, that is: $\mu=\mu(K), \quad \sigma=\sigma(K)$, and its functional relationship can be determined by equation (10) and equation (12). In this case, the first equation in the equations (8) can be regarded as a nonlinear equation with one variable of the shape parameter $K$, which can be written in the form of a function as follows:

$$
\begin{aligned}
& y=f(K)=\frac{n}{K}+\sum_{i=1}^{n} \ln \left(\frac{\mu(K)-x_{i}}{\sigma(K)}\right) \\
& -\sum_{i=1}^{n}\left\{\left(\frac{\mu(K)-x_{i}}{\sigma(K)}\right)^{K} \ln \frac{\mu(K)-x_{i}}{\sigma(K)}\right\}
\end{aligned}
$$

It should be noted that for equation (11) and equation (13), it is possible that there is no zero point of the function. In this case, the above method will not be feasible. In order to improve the method, the point corresponding to the minimum absolute value of the function can be taken as the zero point of equation (11) and equation (13). Generally, this point isn't the best estimate of the parameters of Weibull distribution.

Using the above algorithm process to solve the maximum likelihood parameter estimation problem of Gumbel distribution and Frechet distribution, that is, using the maximum likelihood method to solve the following two groups of two or three simultaneous 
transcendental equations, we can obtain the following probability model:

(1) Extreme value of wind speed distribution type I

$$
\left\{\begin{array}{l}
\mu=-\sigma \ln \left[\frac{1}{n} \sum_{i=1}^{n} \exp \left(-\frac{x_{i}}{\sigma}\right)\right] \\
\sum_{i=1}^{n} x_{i} \exp \left(-\frac{x_{i}}{\sigma}\right)-(\bar{x}-\sigma) \sum_{i=1}^{n} \exp \left(-\frac{x_{i}}{\sigma}\right)=0
\end{array}\right.
$$

(2)

Extreme value of wind speed distribution

type II

$$
\left\{\begin{array}{l}
\sigma=\left[\frac{1}{n} \sum_{i=1}^{n}\left(x_{i}-\mu\right)^{-K}\right]^{-\frac{1}{K}} \\
\sum_{i=1}^{n}\left(x_{i}-\mu\right)^{-(K+1)}-\frac{K+1}{n K} \sum_{i=1}^{n}\left(x_{i}-\mu\right)^{-K} \sum_{i=1}^{n}\left(x_{i}-\mu\right)^{-1}=0 \\
\frac{n}{K}+n \ln \sigma-\sum_{i=1}^{n} \ln \left(x_{i}-\mu\right)-\sum_{i=1}^{n}\left[\left(\frac{\sigma}{x_{i}-\mu}\right)^{K} \ln \left(\frac{\sigma}{x_{i}-\mu}\right)\right]=0
\end{array}\right.
$$

(3) Extreme value of wind speed distribution type III

$$
\left\{\begin{array}{l}
\sigma=\left[\frac{1}{n} \sum_{i=1}^{n}\left(\mu-x_{i}\right)^{K}\right]^{\frac{1}{K}} \\
\frac{K-1}{n K} \sum_{i=1}^{n}\left(\mu-x_{i}\right)^{-1} \sum_{i=1}^{n}\left(\mu-x_{i}\right)^{K}-\sum_{i=1}^{n}\left(\mu-x_{i}\right)^{K-1}=0 \\
\frac{n}{K}+\sum_{i=1}^{n} \ln \left(\frac{\mu(K)-x_{i}}{\sigma(K)}\right)-\sum_{i=1}^{n}\left\{\left(\frac{\mu(K)-x_{i}}{\sigma(K)}\right)^{K} \ln \frac{\mu(K)-x_{i}}{\sigma(K)}\right\}=0
\end{array}\right.
$$

\subsection{Probability weighted moment method}

For the final calculation comparison, we also briefly introduce the probability weighted moment method [22], which is a method to obtain parameter estimation by using the probability weighted moment of the sample to estimate the probability weighted value of the population. Let the probability weighted moment value to $k_{r}$, namely $k_{r}=E\left[\mathrm{xF}(\mathrm{x})^{r}\right]$ (here $\left.\mathrm{r}=0,1,2\right)$, then the unbiased estimation of the first third order probability weighted moment value $d_{0}, d_{1}$, and $d_{2}(\mathrm{r}=0,1,2)$ are

$$
\begin{aligned}
& \left\{\begin{array}{l}
k_{0}=\bar{x}=\frac{1}{n} \sum_{i=1}^{n} x_{i} \\
k_{1}=\sum_{j=1}^{n-1} \frac{(\mathrm{n}-\mathrm{j}) x_{j}}{n(\mathrm{n}-1)(\mathrm{n}-2)} \\
k_{2}=\sum_{j=1}^{n-2} \frac{(\mathrm{n}-\mathrm{j})(\mathrm{n}-\mathrm{j}-1) x_{j}}{n(\mathrm{n}-1)(\mathrm{n}-2)} \\
\hat{\sigma}=2.8854 k_{1}-1.4427 k_{0}
\end{array}\right. \\
& \hat{\mu}=1.8327 k_{0}-1.6654 k_{1}
\end{aligned}
$$

The probability weighted moment method can also be used to estimate the parameters of extremum II and extremum III distribution. According to the generalized extreme value distribution, the shape parameter, scale parameter and position parameter are defined as fellows:

$$
\hat{K}=7.859 c+2.9554 c^{2}
$$

$$
\begin{gathered}
\hat{\sigma}=\frac{\left(2 k_{1}-k_{0}\right) \hat{K}}{\Gamma(1+\hat{K})\left(2-2^{-\hat{K}}\right)} \\
\hat{\mu}=k_{0}+\frac{\hat{\sigma}[\Gamma(1+\hat{K})-1]}{\hat{K}}
\end{gathered}
$$

The parameters $c=\frac{2 k_{1}-k_{0}}{3 k_{2}-k_{0}}-\frac{\ln 2}{\ln 3}, k_{0}$, and $k_{1}$ are shown in equation (17).

\section{Numerical experiments}

\subsection{Wind data}

The wind speed samples used in this paper are from the data of Baoshan meteorological station in Shanghai. The general situation of wind speed stations around the project site is shown in Table 1. The meteorological station records the maximum wind speed per hour (10 min average time interval) at the height of $10 \mathrm{~m}$ for 33 years from January 1, 1975 to December 31, 2007, with a total of $33 * 365 * 24=289080$ data and corresponding wind direction. The wind direction distribution of wind speed sampling data (wind rose diagram) is shown in Figure 1.

Table 1. Overview of wind speed stations around the project

\begin{tabular}{|c|c|c|c|c|c|c|}
\hline $\begin{array}{c}\text { Station } \\
\text { name }\end{array}$ & Region & $\begin{array}{c}\text { longitude } \\
(\mathbf{o})\end{array}$ & $\begin{array}{c}\text { latitude } \\
(\mathbf{o})\end{array}$ & $\begin{array}{c}\text { Altitude } \\
(\mathbf{m})\end{array}$ & $\begin{array}{c}\text { Observation } \\
\text { time }\end{array}$ & $\begin{array}{c}\text { Site } \\
\text { type }\end{array}$ \\
\hline $\begin{array}{c}\text { Baoshan } \\
\text { weather } \\
\text { station }\end{array}$ & Shanghai & 121.48 & 31.41 & 3.86 & $1975-2007$ & typeB \\
\hline
\end{tabular}

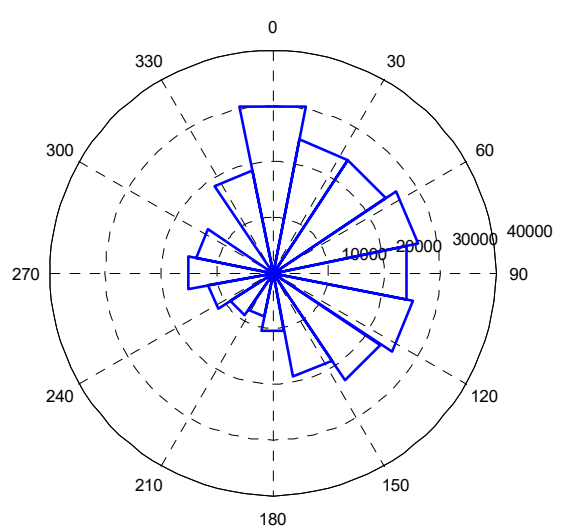

Fig 1. Wind rose chart of Baoshan weather station

The sampling method used in this paper is consistent with the sampling method in literature [7] (stage extreme value method). In general, the daily maximum wind speed samples are extracted from the whole wind speed data for analysis. There are 24 wind speed samples in a day, that is, the daily extreme wind speed of the event is taken as the sample, with a total of 11888 data, that is, $x_{i}$, $i=1,2,3, \ldots . ., 11888$. 


\subsection{Numerical calculation and result analysis}

For the sampled wind speed data, this algorithm is used to calculate the parameter estimation of scale parameter $\sigma$, position parameter $\mu$ and shape parameter $K$.

The numerical method of maximum likelihood estimation for three parameter Weibull distribution can be summarized into the following steps:

Step 1: set the value range $\left(K_{1}, K_{2}\right)$ of shape parameters $K$. In general engineering problems, there is $1<K<10$, so $K_{1}=1, \quad K_{2}=1$ can be used.

Step 2: calculate position parameter $\mu$ and scale parameter $\sigma$ by dichotomy corresponding to $K_{1}$ and $K_{2}$, and calculate the function values of formula (13), and record them as $y_{1}$ and $y_{2}$.

Step 3: Let $K_{3}=\frac{K_{1}+K_{2}}{2}$, then calculate its corresponding position parameter $\mu$ and scale parameter $\sigma$, and the function value $y_{3}$ of equation (13); if the target value of $\left|y_{3}\right|$ has been reached (whether the ideal target is reached can be judged by relative or absolute error), stop the program, otherwise continue.

Step 4: if $y_{1} y_{3}<0$, then $K_{1} \Leftarrow K_{1}, K_{2} \Leftarrow K_{3}$; if $y_{1} y_{3}>0$, then $K_{1} \Leftarrow K_{3}, K_{2} \Leftarrow K_{2}$, and go to step (2).

According to the above steps, the matlab program of the whole process of data processing and analysis is designed. In addition to the algorithm mentioned in the first section of this paper, the traditional method for solving nonlinear equations is a mixture of dichotomy, quadratic inverse interpolation and secant method. The traditional numerical method can also be used to solve the three parameter estimates. Its advantage is that the calculation results are accurate and can be used as a comparison standard, but the calculation process uses a variety of complex calculation methods, which is very cumbersome. In this paper, the traditional method of solving nonlinear equations, the numerical method of maximum likelihood estimation and the probability weighted moment method are used to estimate the parameters of the wind speed probability model based on the above wind speed sampling samples, and the numerical results are compared. The calculation results are shown in Table 2.

Table 2. Parameter estimation results and comparison

\begin{tabular}{l|c|c|c|c}
\hline \multicolumn{2}{c|}{ parameter } & $\begin{array}{c}\sigma \\
\text { (scale) }\end{array}$ & $\begin{array}{c}\mu \\
\text { (position) }\end{array}$ & $\begin{array}{c}K \\
\text { (shape) }\end{array}$ \\
\hline $\begin{array}{c}\text { algorithm } \\
\begin{array}{c}\text { algorithm } \\
\text { proposed } \\
\text { in this paper }\end{array}\end{array}$ & extremumI & 1.2750 & 5.8938 & \\
\hline & extremumII & 4.2838 & 1.9998 & 0.2000 \\
\cline { 2 - 5 } & extremumIII & 17.2150 & 23.1650 & 13.1719 \\
\hline $\begin{array}{c}\text { traditional } \\
\text { algorithm }\end{array}$ & extremumI & 1.2750 & 5.8938 & \\
\hline & extremumII & 4.9100 & 1.8000 & 4.6932 \\
\hline & extremumIII & 11.2780 & 17.2407 & 8.7185 \\
\hline $\begin{array}{c}\text { probability } \\
\text { weighted }\end{array}$ & extremumI & 1.8623 & 10.7632 & \\
\hline
\end{tabular}

\begin{tabular}{l|l|l|l|l}
\hline $\begin{array}{c}\text { moment } \\
\text { method }\end{array}$ & & & & \\
\hline & extremumII & 12.9673 & 13.7314 & 38.4167 \\
\hline & extremumIII & 13.4275 & 14.1743 & 39.8143 \\
\hline
\end{tabular}

It can be seen from the above calculation results that: (1) for the extreme value type I distribution, the results of the numerical solution method proposed in this paper is same as those of the traditional numerical solution; for the extreme value type II, the results of the numerical solution method in this paper are also same as those of the traditional numerical solution, but there is difference between two solutions for the extreme value type III. (2) It is obvious that the numerical method proposed in this paper is more accurate than the probability weighted moment method. (3) Compared with the weighted moment method, the results are more accurate. Using this algorithm can achieve the accuracy of the traditional algorithm, but the calculation process is simpler and faster than the traditional algorithm.

\section{Conclusion}

In this paper, the problem is simplified by means of order reduction and the ternary equations are transformed into binary equations. Firstly, the solutions of the dualistic simultaneous equations are solved. Then, the scale parameters and position parameters are expressed as the functions of shape parameters, so that the maximum likelihood function becomes the univariate function of shape parameters, and then the shape parameters are estimated. In this way, the likelihood estimation of the parameters of the three parameter Weibull distribution is given by combining the dichotomy method A set of numerical methods for solving simultaneous transcendental equations is established. Compared with other algorithms, this method does not need to give the initial value. The algorithm is stable, highly accurate and convergent fastly. In addition, in order to improve the calculation accuracy and convergence speed to a greater extent, other efficient numerical algorithms can be used to replace dichotomy (dichotomy must be used in a monotone interval, otherwise the program operation will be in dead loop), which shows the flexibility of the method and is convenient for engineering application.

\section{References}

1. Writing group, Guide for wind resistance design of Highway Bridges, People's Communications Press, Beijing, China, (1996).

2. E. SIMIU, R.H. SCANLAN. Effect of wind on structure. Liu Shangpei, Xiang Haifan and Xie Jiming, translation. Shanghai: Tongji University Press, (1992).

3. TADELE AKEBA DIRIBA et al. Modelling dependence structures of extreme wind speed using bivariate distribution: a bayesian approach. Environmental and Ecological Statistics,27:319-362, (2020). 
4. B.YAO, Y. QUAN, Study on the analysis method of extreme wind speed in mixed climate areas. Engineering Mechanics,35(5) :86-92, (2018).

5. Q.Li, Extreme Wind Speed Estimation in mixed wind climates and study on analysis models of wind-induced responses of a tall building, $\mathrm{PhD}$ Thesis, Zhejiang University, (2018).

6. C. Lou, F. Dong, Research on extreme wind speed forecasting method of buildingstructure based on Bayesian theory,46(s1) :986-988, (2016).

7. Y.Ge, Research on wind-induced reliability and its application of bridge structure, Tongji university, Shanghai, (1998).

8. H.C.S THOM, Distributions of extreme winds in the united states. Journal of the Structural Division, Proceedings of the ASCE, 86 (4):11-24 (1960).

9. E. SIMIU, J. CHANGERYM, J.J. FILLIBEN. Extreme wind speeds at 129 airport station. Journal of the Structural Division, 106 (4): 809 817, (1980).

10. F. Dong, J.Cheng, Wind speed prediction of extreme value type I distribution basedon the Bayes method, Journal of Harbin institute of technology, 49(3):9397, (2017).

11. B.ZHANG, Y.QUAN, M.GU, A probabilistic method for estimating extreme wind pressure onstructures considering direction effect of extreme wind speed, 39(4): 1-7, (2018).

12. Z.Duan, J.Ou, D.Zhou, The optimal probabilistic distribution for extreme wind speed, China civil engineering journal , 35(5):11-16,(2002).

13. J. Deng, D. Gu, X. Li, Parameters and quantile estimation for fatigue life distributionusing probability weighted moments,21(5):609-613, (2004).

14. A.A. Bartolucci, K.P. Singh, A. D. Bartolucc, et al., Applying medical survival data to estimate the three-parameter Weibull distribution by the method of probability weighted moments. Mathematics and Computers in Simulation, 48(4-6) : 385-392, (1999) .

15. K. E. AHMAD . Modified weighted least-squares estimators for the three-parameter weibull distribution, Applied Mathematics Letters, 7(5) :53$56,(1994)$.

16. M.Fu, Z.Cao, An optimization method of correlation coefficient for determining a three-parameter Weibull distribution,11(7): A323-A327, (1990) .

17. H.Fei, D.Chen,Parameter Estimation of the ThreeParameter Weibull Distribution, Journal of Shanghai Normal University, 25(2) :1-8, (1996).

18. H.Z. QIAO, C.P. TSOKOS, Estimation of the three parameter Weibull probability distribution. Mathematics and Computers in Simulation, 39(12): 173-185, (1995) .

19. J.H. GOVE, S.E. FAIRWEATHER, Maximumlikelihood estimation of Weibull function parameters using a general interactive optimizer and grouped data. Forest Ecology and Management, 28(1) :61-69, (1989) .

20. Y. Qu, C. Zhang, S. Yan, Estimating the parameters for the three-parameter Weibull distribution, 45(3) : 374-375, (1987).

21. H.Wang, Z.Li, R.Lin, Maximum likelihood estimation for three-parameter Weibull distribution based on wear out fault, 25(5): 39-42, (2004).

22. JRM. HOSKING, J.R. WALLIS, E.F. WOOD, Estimation of the generalized extreme value distribution by the method of probability-weighted moments. Technom-etrics,27(3): 251-261, (1985). 